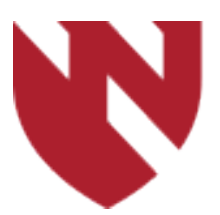

September 2020

\title{
In-Hospital Medication Delivery in Status Epilepticus: Targeting the Delays in Drug Dispensing and Administration
}

\author{
Navnika Gupta \\ University of Nebraska Medical Center \\ Hae Y. Baang \\ University of Nebraska Medical Center \\ Nicholas Swingle \\ University of Nebraska Medical Center \\ Katharine Reisbig \\ University of Nebraska Medical Center \\ Kayli A. Bendlin \\ University of Nebraska Medical Center
}

See next page for additional authors

Tell us how you used this information in this short survey.

Follow this and additional works at: https://digitalcommons.unmc.edu/gmerj

Part of the Higher Education Commons, and the Medicine and Health Sciences Commons

\section{Recommended Citation}

Gupta, N., Baang, H. Y., Swingle, N., Reisbig, K., Bendlin, K. A., Coleman, S. A., Goetschkes, K. R., , Taraschenko, O. In-Hospital Medication Delivery in Status Epilepticus: Targeting the Delays in Drug Dispensing and Administration. Graduate Medical Education Research Journal. 2020 Sep 29; 2(1). https://digitalcommons.unmc.edu/gmerj/vol2/iss1/52 
In-Hospital Medication Delivery in Status Epilepticus: Targeting the Delays in Drug Dispensing and Administration

\section{Creative Commons License}

\section{(c) (1) $\odot \Theta$}

This work is licensed under a Creative Commons Attribution-Noncommercial-No Derivative Works 4.0 License.

Authors

Navnika Gupta, Hae Y. Baang, Nicholas Swingle, Katharine Reisbig, Kayli A. Bendlin, Scott A. Coleman, Kelly R. Goetschkes, and Olga Taraschenko 


\section{In-Hospital Medication Delivery in Status Epilepticus: Targeting the Delays in Drug Dispensing and Administration \\ Navnika Gupta' ${ }^{1}$, Hae Y. Baang ${ }^{1}$, Nicholas Swingle, Katharine Reisbig ${ }^{2}$, Kayli A. Bendlin², Scott A. Coleman², Kelly R. Goetschkes ${ }^{1}$, Olga Taraschenko $^{1}$ 'University of Nebraska Medical Center, Department of Neurological Sciences \\ 2University of Nebraska Medical Center, College of Pharmacy}

Mentor: Olga Taraschenko

Program: Neurological Sciences

Type: Original Research

Background: We have previously shown that the delivery of the first, second and third order medications for nonconvulsive status epilepticus (NCSE) was delayed at the University of Nebraska Medical Center (UNMC), a level 4 epilepsy center. We established that the delay in medication delivery was caused by untimely recognition of seizures and prolonged medication order processing. To overcome the latter barrier and streamline the completion of medication orders, we examined the points of specific delays within the inpatient pharmacy. We also engaged pharmacy managers to provide staff education and support the placement of commonly used anticonvulsants in the Omnicell medication dispensing system on the hospital floors.
Methods: We implemented the Omnicell medication dispensing system for the use of intravenous levetiracetam (LEV) and lacosamide (LCM) on the neurology floor, introduced SE order set and SE response protocol. Retrospective and prospective chart analysis was performed to compare the times from the order to administration of all anticonvulsants following the implementation of these measures with the corresponding times during the preceding three-year period for all patients with NCSE; the anesthetic medications were excluded.

Results: A total of 77 and 38 orders for the first, second, and third order anticonvulsants for NCSE were placed prior to and following the implementation of floor-based dispensing system, respectively. Of them, intravenous LEV and LCM were ordered in 24 (31.2\%) and $14(36.8 \%)$ of instances prior to and after the improvement, respectively; the proportions of these orders were similar in two groups ( $\mathrm{p}=0.54$; Pearson's chi- squared test). The median latencies to the administration of all anticonvulsants prior and following the implementation of the Omnicell system were 34 (IQR 18-58) and $21 \mathrm{~min}$ (IQR $12-45)$, respectively ( $\mathrm{p}=0.18$, Mann-Whitney test). The median time to the administration of LEV and LCM have decreased from 57 (IQR 41-99) to $42 \mathrm{~min}$ (IQR 31-105) after the introduction of the Omnicell dispensing system and staff education ( $\mathrm{p}=0.41$; MannWhitney test).

Conclusion: The delivery of treatment for NCSE may be streamlined by the education of pharmacy staff and closer proximity of the medications commonly used in acute seizure emergencies to the patients' bedside. To further improve the effectiveness of care for patients with NCSE, we provided the education to the fellows from the Critical Care services and plan to implement an educational course for nurses from neurology floor and intensive care units.

https://doi.org/10.32873/unmc.dc.gmerj.2.1.050

\section{Functional MRI Changes in Ischemic Stroke Patients Following Mechanical Thrombectomy}

Praveen Hariharan', Marco A. Gonzalez-Castellon', Connor J. Phipps ${ }^{1}$, David E. Warren ${ }^{1}$

${ }^{1}$ University of Nebraska Medical Center, Department of Neurological Sciences

Mentor: Marco A. Gonzalez-Castellon

Program: Neurological Sciences

Type: Original Research

Background: Recent evidence in stroke research suggests that functional connectivity measures such as modularity assessed by resting state functional MRI (rs-fMRI) may provide insights in predicting prognosis and may help characterize outcome differences associated with mechanical thrombectomy (MT). We measured brain network modularity longitudinally in MT-treated ischemic stroke patients.

Methods: This study was approved by Institutional Review Board at the University of Nebraska Medical Center. MT-treated stroke patients were recruited. A 3T Siemens Prisma MRI scanner was used to collect data with a protocol adapted from the Human Connectome Project (HCP). The protocol included structural measures as well as rs-fMRI measures (multiband EPI). Images were acquired within first two weeks and three months after stroke. rs-fMRI data were processed with HCP pipelines. Network modularity was measured using a previously reported cortical parcellation.

Results: We recruited three patients $(1 \mathrm{~F} / 2 \mathrm{M}$; mean age $=56$ years). Average NIHSS was 11. Two were treated with IV thrombolysis plus MT and one was treated with MT. Two strokes had significant cortical infarction; the other was subcortical. Mean door-toneedle time was 35 min.; mean reperfusion time was $201 \mathrm{~min}$. Modularity measures showed that patients with cortical infarcts exhibited increased modularity between time points, while the subcortical infarct exhibited decreased modularity.

Conclusion: Increases in brain network modularity over time in two out of three MT-treated patients may reflect restoration or reorganization of brain networks to support normal brain function. Longitudinal cohort studies of stroke could help us better understand functional brain network disruption, track recovery, guide stroke interventions, and improve prognostication.

https://doi.org/10.32873/unmc.dc.gmerj.2.1.051 